\title{
Research on Photovoltaic Control Strategy Based on Particle Swarm Optimization Algorithm
}

\author{
Huaizhong Chen \\ Zhejiang Industry Polytechnic College, Shaoxing, China \\ Chz702@163.com
}

Keywords: PSO; Photovoltaic Power; MPPT; Algorithm

\begin{abstract}
Aiming at the disadvantage of low accuracy of the photovoltaic maximum power point constant voltage method, a photovoltaic MPPT algorithm based on particle swarm optimization algorithm (PSO) is proposed. Based on the analysis and research of the photovoltaic array maximum power point tracking algorithm, the traditional MPPT control is optimized . When the system deviates from the maximum power point, the particle swarm optimization is used to make the maximum power control, which makes the MPPT to ensure the tracking speed and improve the tracking accuracy. Finally, the algorithm is simulated by Matlab, and the results show that the control system can track the maximum power point quickly.
\end{abstract}

\section{Introduction}

The cost of photovoltaic panels has been very high, but the low conversion efficiency of photovoltaic power generation to make large-scale development, we must improve the efficiency of power conversion. The photovoltaic power generation system can ensure the maximum power output under different environmental conditions, the maximum power point tracking (MPPT) algorithm has become a research hotspot. Commonly used traditional methods such as constant pressure method, disturbance observation method, and conductance increment method, etc., the disturbance observation method is simple and easy to be realized, but there is oscillation near the maximum power point. The method has high precision, but it may happen when the environment changes. Based on the above research, this paper proposes a MPPT algorithm based on PSO. Not only can track the changes of the external environment, but also have good stability, which can effectively reduce the oscillation of the system output voltage, and improve the utilization rate of photovoltaic cells. The algorithm provides a research way to reduce the cost of MPPT device and reduce the volume of the device[1,2].

\section{Photovoltaic MPPT Control Principle}

At the same intensity of environmental temperature, light conditions, photovoltaic cells can be seen as a linear voltage source. The photovoltaic cell circuit is simplified as a constant voltage source in series with a resistor to the load, so the power supply circuit can be simplified as shown in Fig.1. Among them, $R_{P V}$ is the resistance of photovoltaic cells, equivalent to photovoltaic battery series battery, shunt resistance; $\mathrm{R}_{\mathrm{L}}$ is load resistance; $\mathrm{i}_{\mathrm{L}}$ is load current; $\mathrm{U}$ is voltage source voltage[4].

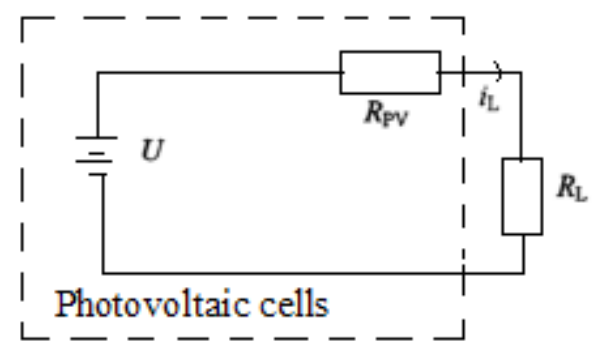

Figure 1. Principle of maximum power point matching

From the image above shows that the load power is: 


$$
P_{L}=i_{L}^{2} R_{L}=\left(\frac{U}{R_{L}+R_{P V}}\right)^{2} R_{L}
$$

The formula of the $\mathrm{R}_{\mathrm{L}}$ derivative can be obtained:

$$
\frac{d_{P_{L}}}{d_{R_{L}}}=\frac{U^{2}\left(R_{P V}-R_{L}\right)}{\left(R_{L}+R_{P V}\right)^{2}} R_{L}
$$

If the reciprocal of the above is zero, we can obtain follow equation:

$$
R_{P V}=R_{L}
$$

That is, when the formula 3 is established, the load power reaches the maximum value. For the photovoltaic power supply circuit on the circuit load, when the load resistance and the resistance value is equal to the maximum output power of photovoltaic cell. Due to environmental factors and load changes, the output impedance of PV cells is not matched with the load impedance, which can reduce the power output of photovoltaic power generation system, and result in the loss of energy[4,5].

At present, the main method to solve this problem is to use the maximum power point tracking technology. The switch between the photovoltaic cell and the load is added to the switch circuit, and the real-time match control of the load impedance and the internal resistance of the photovoltaic cell is realized by the MPPT controller, so that the maximum power of the photovoltaic cell is always output.

\section{MPPT Control Based On PSO Algorithm}

Basic Principles of PSO Algorithm. Particle swarm optimization (swarm optimization particle) algorithm is a new global optimization method based on swarm intelligence theory. Swarm intelligence guidance and optimization search through cooperation and competition among particles in a group. It is proposed by Kennedy and Eberhart that the algorithm is simple and has less parameter and has the ability of global optimization. It has been applied in many fields. In the PSO algorithm, the individual is gradually moved to a better area according to the degree of adaptation of each particle to the environment. The final search finds the optimal solution to the problem[6,7].

In the PSO algorithm, the I particles in the $\mathrm{K}$ flight at a speed of $\mathrm{v}_{\mathrm{i}}(\mathrm{k})$ flight to the position of $\mathrm{Si}$ in the search space $S_{i}(k)$, in the $k+1$ flight speed of $v_{i}(k+1)$ is to update previous speed according to the optimal value $\mathrm{v}_{\mathrm{i}}(\mathrm{k})$. One is flying to the optimal particle itself at the moment to find the value, namely the individual optimal value of pbest $\mathrm{i}_{\mathrm{i}}$; the other is the whole particle swarm flight to find the optimal moment value, which is all the optimal value of gbest. Get $v_{i} \quad(k+1)$, the first $k+1$ flight to the location of $S_{i}(k+1)$ can be updated by $v_{i}(k+1)$. The velocity, the position, the individual optimal value, the global optimum value by the formula (1), (2), (3) carries on the renewal:

$$
\begin{aligned}
& v_{i}(k+1)=\omega v_{i}(k+1)+c_{1} r_{1}\left[\text { pbest }_{i}-s_{i}(k)+c_{2} r_{2}\left[\text { pbest }-s_{i}(k)\right]\right. \\
& s_{i}(k+1)=s_{i}(k)+v_{i}(k+1) \\
& \text { pbest }_{i}=\max \left\{f\left(s_{i}(k+1)+f\left(\text { pbest }_{i}\right)\right\}\right. \\
& \text { gbest }_{i}=\max \left\{f\left(\text { gbest }_{1}\right), f\left(\text { gbest }_{2}\right), \ldots, f\left(\text { gbest }_{n}\right)\right\}
\end{aligned}
$$

Among them: $\mathrm{i}=1,2, \ldots . \mathrm{n}, \mathrm{n}$ is the number of particles in the population. $\mathrm{W}$ is the inertia weight; $\mathrm{c}$ is the learning factor; $\mathrm{r}$ is the random number of random distribution; The objective function $\mathrm{f}$ is the output power; The $\mathrm{S}$ is the duty cycle; The $\mathrm{V}$ is the change of the duty cycle. 
The idea of distributed MPPT control algorithm based on PSO is that the output voltage of multiple modules as a multi-dimensional variable, the total power output of the system as the objective function. Using particle swarm optimization algorithm to find the maximum value of the total output power. It is also found that each module of the maximum power point, and then through the conventional method of constant voltage and the output voltage of solar panels at the maximum power point, so as to realize the maximum power of distributed photovoltaic power system control.

Inertia Weight Setting. Considering the effects of inertia weight PSO global and local search ability, large inertia factor is conducive to jump out of the local minimum and improve the tracking speed. The inertia weight less conducive to local search precision, improve the tracking accuracy[8].

According to this principle, the formula of the inertia weight $\mathrm{k}$ with the iteration number $\mathrm{w}$ is as follows:

$\omega=0.82-0.045 k k<12$

$\omega=0.35 \quad k \geq 12$

The influence of the velocity of the particle on the current velocity is described by the inertia weight $\mathrm{W}$. The global and local optimization ability of the standard particle swarm optimization algorithm can be adjusted by controlling the size of the particle swarm. The greater the $\mathrm{W}$, the stronger the global search ability, the weaker the local search ability, the local search ability is enhanced, and the global search ability is weakened[9].

Algorithm Iteration Stop Setting. In order to enable the algorithm to capture the changes in the external environment, when the iteration to the first $\mathrm{k}, \mathrm{N}$ particle output voltage with respect to the variance of the global optimal voltage at this time to meet $\mathrm{D}(\mathrm{k})<1(\mathrm{k})$, then the iterative algorithm. The duty cycle is obtained at the end of the global optimal value of constant output. The formula of $\mathrm{D}(\mathrm{k})$ is:

$$
D(K)=\frac{1}{n}=\sum_{i=1}^{n}\left(U_{i}-U_{\text {gbest }}\right)^{2}
$$

MPPT particle swarm optimization algorithm flow:

In the maximum power tracking control of photovoltaic cells, the whole algorithm is divided into 2 parts: a part of the particle swarm optimization and the other part is the maximum power tracking part. In the PSO algorithm, the potential solution of each optimization problem which is the maximum power point voltage is a "particle" in the search space, and all the particles have an adaptive value that is determined by the objective function. Specific steps are as follows:

1) Initialization and the determination of the objective function.

Set population quantity and evolution algebra. And the learning factors, weight coefficient and maximum speed and other parameters are initialized. At last, the position and velocity of each particle are initialized.

2) The evaluation of the population in the various parameters of the initialization, the calculation of each particle corresponding to the objective function of the fitness value.

3) Comparison of individual and global fitness and extreme value.

4) Update the particle velocity and position. Update the velocity position of all particles according to above formula

5) Check the end conditions, if the satisfaction, end optimization, the output of the optimal solution; otherwise, $t=t+1 \mathrm{~T}$, go to 2 ), the end conditions for optimization to reach the maximum evolutionary algebra. 


\section{Algorithm Simulation}

Written in PSO Matlab algorithm M files, through the way of programming Simulink and M files to exchange data, and output data to the Matlab soil for space, and ultimately draw the power tracking curve shown in Fig.2.

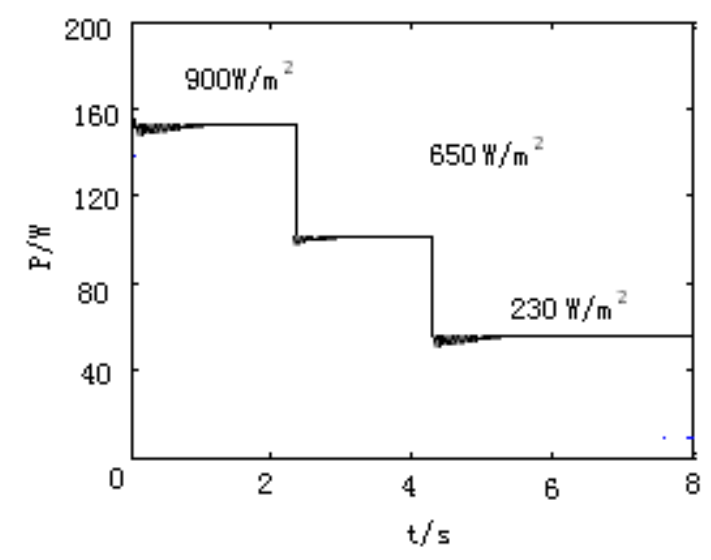

Fig.2 Photovoltaic power tracking curve

It can be seen from the figure 3 that the output voltage of the solar module is quickly adjusted to the voltage value of the maximum power point of the output of the particle swarm. Steady state power is not stable, and the error is very small, which shows that the particle swarm optimization module can find the maximum power point voltage $[11,12]$.

\section{Conclusions}

When the intensity of light is uneven, the output characteristic curve will be more than one peak value. Traditional maximum power algorithm for multi peak MPPT tracking, the tracking results are random. PSO optimization algorithm is used to track the maximum output power of PV array under local shading. The simulation results show that the improved particle swarm algorithm is accurate, stable and steady, steady state power fluctuations, and improve the efficiency of photovoltaic power generation system.

\section{Acknowledgements}

This work is supported by scientific research funded project of Science Technology Department of Zhejiang Province (2015C31128).

\section{References}

[1] B.S.Ubudhl: IEEE Transactions on Sustainable Energy, Vo4.107 (2013), No.1, p.89-98.

[2] H.X. Ma:Power Electronic Technology, Vol.45(2012),No.1,p.73-75.

[3] K.M Tsang, W.L. Chan: Energy Conversion and Management, Vol.70 (2013), No.11, p. 83-89.

[4] I. Kashif , S. Zainal: IEEE Applied Power Electronics Colloquium, Vol.2 (2010), No.2, p.5-9.

[5] J. SONG, Q.XU: Proceeding of the CSU-EPSA, Vol.22 (2012), No.6, p.19-123.

[6] X.Y. LIU, Q.I Xin: Power System Technology, Vol.34 (2010), No.11, p.192-197.

[7] S.M. HUANG, L.S.Lin: Proceeding of the CSU-EPSA, Vol.23 (2010), No.5, p.26-30.

[8] ]P.H.Mellor:IEE Seminar on Electric, Hybrid and Fuel Cell Vehicles, Vol.17(2012),No.8,p.1-5.

[9] Z. Salam: A technological and status review, Applied Energy, Vol.107 (2013), No.15, p.135-148. 
[10]R.Chamnan,T.Tanes: International Conference on Control Automation Systems( Korea,November,2011), p.1391-1395.

[11]M.Masafumi,V.Mummadi:IEEE Transactions on Aerospace and Electronic systems.Vol.47(2011),No.1,p.367-380.

[12] D.Linzen,S.Buller, E. Karden, et al. IEEE Trans on Industry Applications, Vol.41 (2014), No.5, p.1135-1141. 